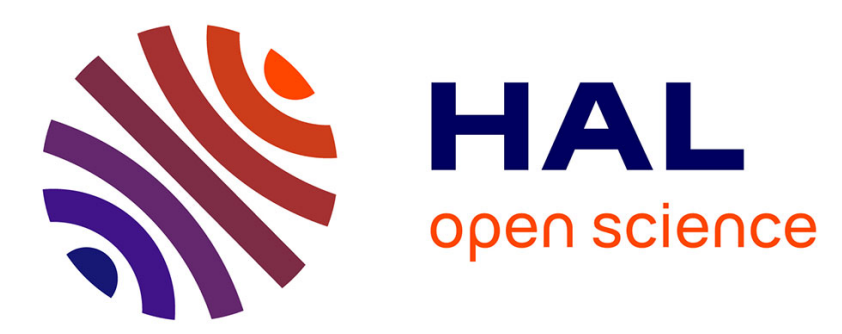

\title{
Simple and efficient approach to the optimization of correlated wave functions
}

Anthony Scemama, Claudia Filippi

\section{To cite this version:}

Anthony Scemama, Claudia Filippi. Simple and efficient approach to the optimization of correlated wave functions. Physical Review B: Condensed Matter and Materials Physics (1998-2015), 2006, 73, pp.241101(R). hal-00180167

\section{HAL Id: hal-00180167 https://hal.science/hal-00180167}

Submitted on 28 Jan 2020

HAL is a multi-disciplinary open access archive for the deposit and dissemination of scientific research documents, whether they are published or not. The documents may come from teaching and research institutions in France or abroad, or from public or private research centers.
L'archive ouverte pluridisciplinaire HAL, est destinée au dépôt et à la diffusion de documents scientifiques de niveau recherche, publiés ou non, émanant des établissements d'enseignement et de recherche français ou étrangers, des laboratoires publics ou privés. 


\title{
Simple and efficient approach to the optimization of correlated wave functions
}

\author{
Anthony Scemama and Claudia Filippi \\ Instituut-Lorentz, Universiteit Leiden, Niels Bohrweg 2, 2333 CA Leiden, The Netherlands
}

(Received 4 May 2006; published 13 June 2006)

\begin{abstract}
We present a simple and efficient method to optimize within energy minimization the determinantal component of the many-body wave functions commonly used in quantum Monte Carlo calculations. The approach obtains the optimal wave function as an approximate perturbative solution of an effective Hamiltonian iteratively constructed via Monte Carlo sampling. The effectiveness of the method, as well as its ability to substantially improve the accuracy of quantum Monte Carlo calculations, are demonstrated by optimizing a large number of parameters for the ground state of acetone and the difficult case of the $1^{1} B_{u}$ state of hexatriene.
\end{abstract}

DOI: 10.1103/PhysRevB.73.241101

PACS number(s): 02.70.Ss, 31.10.+z, 31.25.Qm

Over the last decade, quantum Monte Carlo (QMC) methods have been employed to accurately compute electronic properties of large molecular and solid systems where conventional quantum chemistry approaches are extremely difficult to apply. ${ }^{1} \mathrm{~A}$ crucial step in both the variational (VMC) and the diffusion Monte Carlo (DMC) approach is the construction of the trial wave function $\Psi_{\mathrm{T}}$ which is usually chosen of the Jastrow-Slater form, that is $\Psi_{\mathrm{T}}=\mathcal{J} \Phi$, where $\Phi$ is a small expansion in Slater determinants and $\mathcal{J}$ the positive Jastrow correlation factor.

Although considerable progress has been made (principally using the variance minimization approach ${ }^{2}$ ) in the construction of optimal Jastrow factors, relatively little attention has been given to the optimization of the determinantal part of the wave function. Methods such as Hartree-Fock (HF) or a small-scale configuration interaction (CI) are used as a practical way of constructing the determinantal component, which is generally not reoptimized when the Jastrow factor is added. However, the determinantal part of the wave function solely determines the DMC energy ${ }^{3}$ and often needs to be reoptimized to obtain accurate results. ${ }^{4} \mathrm{~A}$ practical and simple approach to calculate the optimal determinantal component is therefore particularly important to a wide and successful application of QMC methods.

In recent years, several methods to optimize the wave function through energy minimization have been proposed. ${ }^{4-16}$ A direct approach to energy minimization entails computing the gradient and the Hessian of the energy with respect to the desired parameters. The use of an estimate of the Hessian characterized by reduced statistical fluctuations ${ }^{9,10}$ yields a simple and robust optimization algorithm for the Jastrow parameters. However, the Hessian with respect to the orbital parameters in the determinant is affected by higher statistical noise so that devising a stable energy-minimization scheme is more difficult ${ }^{10}$ : using a modified Hessian still leads to large fluctuations, and is, for instance, less stable than the simple stochastic reconfiguration (SR) method ${ }^{11,12}$ so that, during the optimization, one may have to switch to the inefficient SR to retain stability. To date, the most successful method remains the energy fluctuation potential (EFP) method, ${ }^{4,13-16}$ which determines the optimal determinantal component as the solution of an effective Hamiltonian iteratively constructed via Monte Carlo sampling. The approach has been used to optimize the orbitals, ${ }^{4,14}$ and the linear coefficients in front of the determinants, ${ }^{4,15}$ and has been extended to excited states. ${ }^{4}$ The method is very stable and more efficient than the SR approach but quite complex ${ }^{4}$ : the construction of the starting effective Hamiltonian as well as its update are computationally very demanding steps, especially for large systems.

In this Rapid Communication, we propose a simple and efficient optimization method for the determinantal component of the wave function. The approach constructs the optimal wave function via a perturbative scheme based on the EFP method, and only requires easily accessible quantities from the quantum chemical calculation used to set up the starting wave function. The performance of the method is demonstrated on acetone and on the $1{ }^{1} B_{u}$ state of hexatriene, which is particularly challenging for all conventional electronic structure approaches. First, we will briefly review the EFP method.

The energy fluctuation potential method. Let us assume that the trial wave function $\Psi_{\mathrm{T}}$ depends on a set of parameters $\left\{\alpha_{k}\right\}$. The derivatives of the energy with respect to the parameters can be written as

$$
\frac{\partial E}{\partial \alpha_{k}}=2\left\langle\left(E_{\mathrm{L}}-\bar{E}\right)\left(O_{k}-\bar{O}_{k}\right)\right\rangle,
$$

where $\langle\cdot\rangle$ denotes the average with respect to the square of the trial wave function $\left|\Psi_{\mathrm{T}}\right|^{2}$, which can be computed by Monte Carlo sampling. We defined $\bar{E}=\left\langle E_{\mathrm{L}}\right\rangle$ and $\bar{O}_{k}=\left\langle O_{k}\right\rangle$, where

$$
E_{\mathrm{L}}=\frac{\mathcal{H} \Psi_{\mathrm{T}}}{\Psi_{\mathrm{T}}} \quad \text { and } \quad O_{k}=\frac{1}{\Psi_{\mathrm{T}}} \frac{\partial \Psi_{\mathrm{T}}}{\partial \alpha_{k}}
$$

For the optimal parameters, the derivatives of the energy [Eq. (1)] are zero, and the fluctuations of the local energy $E_{\mathrm{L}}$ and of the functions $O_{k}$ are uncorrelated. This means that the energy is stationary if the remaining fluctuations of the local energy cannot be further reduced by adding some combination of the functions $O_{k}$. Hence, the minimization of the energy can be reformulated as a least-squares fit of the fluctuations of the local energy with the functions $O_{k}$, 


$$
\chi^{2}=\left\langle\left(E_{\mathrm{L}}-V_{0}-\sum_{k>0} V_{k} O_{k}\right)^{2}\right\rangle .
$$

A minimization of $\chi^{2}$ with respect to the parameters $V_{k}$ leads to the following set of linear equations for $m>0$ :

$$
\left\langle\Delta E \Delta O_{m}\right\rangle=\sum_{k>0} V_{k}\left\langle\Delta O_{k} \Delta O_{m}\right\rangle
$$

where we have eliminated $V_{0}$ from the other equations, and $\Delta E=E_{\mathrm{L}}-\bar{E}$ and $\Delta O_{m}=O_{m}-\bar{O}_{m}$. Since the left-hand side of these equations corresponds to the derivatives of the energy [Eq. (1)], the fitting parameters $V_{k}$ are zero if, and only if, all the derivatives of the energy are zero. In general, the parameters $V_{k}$ that solve these linear equations will not be equal to zero and can be used to improve the current trial wave function $\Psi_{\mathrm{T}}$.

We focus here on the EFP procedure to optimize the determinantal part $\Phi$ of the trial wave function $\Psi_{\mathrm{T}}$,

$$
\Psi_{\mathrm{T}}=\mathcal{J} \Phi=\mathcal{J} \sum_{i} c_{i} C_{i}
$$

where a spin-adapted configuration state function (CSF) $C_{i}$ is a linear combination of Slater determinants $D_{i}$ of singleparticle orbitals. Let us assume that the starting $\Phi$ is the lowest solution $\Phi_{0}^{(0)}$ of the CI Hamiltonian $\mathcal{H}^{(0)}$,

$$
\mathcal{H}^{(0)}=\sum_{i} E_{i}^{(0)}\left|\Phi_{i}^{(0)}\right\rangle\left\langle\Phi_{i}^{(0)}\right|,
$$

where the states $\Phi_{i}^{(0)}$ span the same space as the CSFs. To obtain the optimal coefficients $c_{i}$ or equivalently the best solution in the basis of the eigenstates of $\mathcal{H}^{(0)}$, we consider the variations of $\Phi$ with respect to the eigenstates other than $\Phi_{0}^{(0)}$,

$$
\Phi=\Phi_{0}^{(0)} \rightarrow \Phi^{\prime}=\Phi_{0}^{(0)}+\sum_{k>0} \delta_{k} \Phi_{k}^{(0)}
$$

so that $O_{k}=\Phi_{k}^{(0)} / \Phi_{0}^{(0)}$. The quantities appearing in the linear equations [Eq. (4)] are sampled from $\Psi_{\mathrm{T}}=\mathcal{J} \Phi_{0}^{(0)}$ and parameters $V_{k}^{(0)}$ are used to construct a new Hamiltonian $\mathcal{H}^{(1)}$ as

$$
\mathcal{H}^{(1)}=\mathcal{H}^{(0)}+\sum_{k>0} V_{k}^{(0)}\left(\left|\Phi_{0}^{(0)}\right\rangle\left\langle\Phi_{k}^{(0)}\right|+\text { h . c . }\right) .
$$

This Hamiltonian is diagonalized, yielding a new set of states, and the procedure is iterated until convergence.

If we also want to optimize the orbitals in the Slater determinants, we can linearize the problem using a so-called super-CI expansion to treat the CI and orbital variations on the same footing: the CSFs occupied in the wave function [Eq. (5)] are augmented by all possible single excitations from the occupied to a set of external orbitals, and the occupied orbitals can be improved by using the natural orbitals of the CI wave function in this augmented space. ${ }^{17}$ The EFP scheme to optimize the determinantal component via a super-CI approach ${ }^{4}$ is quite complex. The matrix elements of the starting super-CI Hamiltonian must be extracted from the available quantum chemical code, rendering the setup of the approach highly nontrivial. ${ }^{18}$ Moreover, as the number of singly excited CSFs increases with the number of CSFs in the reference wave function and with the size of the basis (i.e., with the number of available external orbitals), both the setup and the update steps quickly become computationally too demanding. ${ }^{19}$

A simplified EFP approach. The basic idea of the scheme proposed here is to avoid the explicit construction of the EFP Hamiltonian by solving the problem perturbatively. For the example given above, we obtain an improved wave function [Eq. (7)] not by diagonalizing the Hamiltonian [Eq. (8)], but approximately, to first order in the perturbation given by the corrections $V_{k}^{(0)}$,

$$
\Phi=\Phi_{0}^{(0)} \rightarrow \Phi^{\prime}=\Phi_{0}^{(0)}-\sum_{k>0} \frac{V_{k}^{(0)}}{E_{k}^{(0)}-E_{0}^{(0)}} \Phi_{k}^{(0)} .
$$

It is simple to show that, in the absence of the Jastrow factor, this is indeed the perturbative solution for $\mathcal{H}-\mathcal{H}^{(0)}$ since $V_{k}^{(0)}=\left\langle\Phi_{k}^{(0)}\left|\mathcal{H}-\mathcal{H}^{(0)}\right| \Phi_{0}^{(0)}\right\rangle$. This perturbative EFP scheme can also be viewed as a generalization of the SR method ${ }^{11,12}$ which yields an improved wave function by applying the operator $\Lambda-\mathcal{H}$ to the current state and projecting the result onto the space defined by the parametrization. The SR wave function is constructed as in Eq. (9) but all the changes are scaled by the same energy denominator while the EFP approach scales each correction appropriately, achieving significantly faster convergence. ${ }^{4}$

Estimate of the energy denominators. The perturbative solution of the EFP problem requires the knowledge of the energy denominators appearing in Eq. (9), which can be easily estimated with the use of quantities readily available from the quantum chemical calculation used to set up the starting wave function. We will first consider the optimization of the orbital parameters.

The simplest case is given by a trial wave function constructed from a single closed-shell determinant as $\Psi_{\mathrm{T}}=\mathcal{J} D_{0}$, where $D_{0}$ is a determinant of Hartree-Fock or density functional Kohn-Sham orbitals which we want to reoptimize in the presence of the Jastrow factor. We assume here that we have chosen HF orbitals but all the following considerations equally apply to the case of density functional orbitals. The set of occupied and virtual HF orbitals, and the atomic basis on which they are expanded, span the same space so that we can express the variations of one orbital with respect to the expansion coefficients in terms of the variations with respect to the other orbitals. To optimize the $M$ occupied orbitals $\varphi_{i}$ in $D_{0}$, we only need to consider the variations with respect to the $N-M$ virtual orbitals as

$$
\varphi_{i} \rightarrow \varphi_{i}^{\prime}=\varphi_{i}+\sum_{j=M+1}^{N} c_{i j} \varphi_{j},
$$

where we only mix orbitals of the same symmetry. The corresponding first-order change in the wave function is

$$
\Psi_{\mathrm{T}} \rightarrow \Psi_{\mathrm{T}}^{\prime}=\mathcal{J} D_{0}+\sum_{i=1}^{M} \sum_{j=M+1}^{N} c_{i j} \mathcal{J} C_{0}^{i \rightarrow j},
$$

where $C_{0}^{i \rightarrow j}=D_{0}^{i \alpha \rightarrow j \alpha}+D_{0}^{i \beta \rightarrow j \beta}$ is the CSF of the two determinants obtained by substituting the orbital $\varphi_{i}$ with orbital $\varphi_{j}$ 


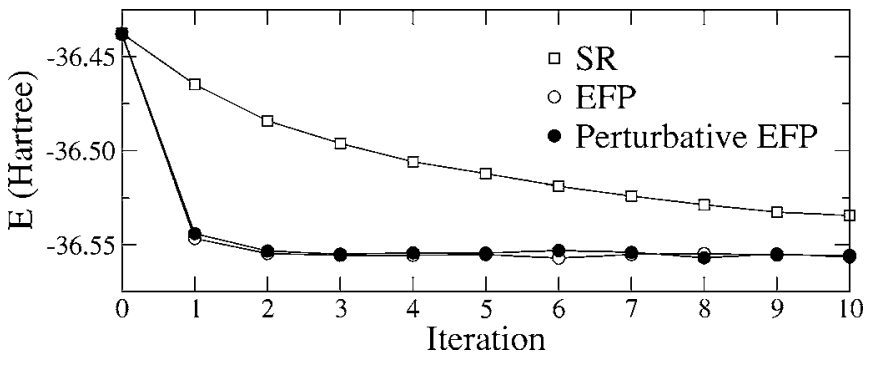

FIG. 1. Convergence of the VMC energy of acetone with the perturbative EFP, the EFP, and the SR method to optimize the 473 orbital parameters. The starting determinantal component is from an unconverged HF calculation. The runs are of $16 \times 10^{5}$ steps. The statistical errors are smaller than the symbol size.

for the up and down spins, respectively. It is simple to show that $O^{i \rightarrow j}=C_{0}^{i \rightarrow j} / D_{0}$.

Following the EFP procedure, we sample from $\Psi_{\mathrm{T}}$ the quantities appearing in the linear equations [Eq. (4)] and obtain the corrections $V^{i \rightarrow j}$ corresponding to the functions $O^{i \rightarrow j}$. We can then obtain an improved determinantal component in analogy to Eq. (9) as

$$
\Phi^{\prime}=D_{0}-\sum_{i=1}^{M} \sum_{j=M+1}^{N} \frac{V^{i \rightarrow j}}{\Delta E^{i \rightarrow j}} C_{0}^{i \rightarrow j},
$$

where we assign an energy scale $\Delta E^{i \rightarrow j}$ to the variation corresponding to the single excitation $C_{0}^{i \rightarrow j}$. We now make the key observation that, to first order, this is equivalent to the much simpler step of constructing an improved set of occupied single-particle orbitals as

$$
\varphi_{i}^{\prime}=\varphi_{i}-\sum_{j=M+1}^{N} \frac{V^{i \rightarrow j}}{\Delta E^{i \rightarrow j}} \varphi_{j},
$$

which can be used in a new single-determinant wave function $\Psi_{\mathrm{T}}^{\prime}=\mathcal{J} D_{0}^{\prime}$. We then proceed iteratively by sampling a new set of $V^{i \rightarrow j}$ from the wave function $\Psi_{\mathrm{T}}^{\prime}$ and updating the orbitals as in Eq. (13) until convergence.

To estimate the energy denominators $\Delta E^{i \rightarrow j}$, we note that the starting HF orbitals are canonical orbitals obtained by diagonalizing the Fock matrix so that the corresponding eigenvalues have a physical interpretation via the Koopman's theorem. Minus the eigenvalues of the occupied and the virtual orbitals are an approximation to the ionization potentials (IP) and the electron affinities (EA), respectively. The energy associated with the promotion of one electron from orbital $\varphi_{i}$ to orbital $\varphi_{j}$ can therefore be estimated as the difference IP-EA, namely

$$
\Delta E^{i \rightarrow j} \sim E^{i \rightarrow j}-E_{\mathrm{HF}} \sim \epsilon_{j}-\epsilon_{i}
$$

where $\epsilon_{i}$ and $\epsilon_{j}$ are the eigenvalues corresponding to orbitals $\varphi_{i}$ and $\varphi_{j}$. We do not find it necessary at successive iterations to update the parameters $\Delta E^{i \rightarrow j}$, which are therefore kept fixed at the starting $\mathrm{HF}$ values.

In Fig. 1, we compare the convergence of the proposed method, denoted by "perturbative EFP," with the EFP and the SR methods for the orbital optimization of a single closedshell Jastrow-Slater wave function for the ground state of acetone $\left(\mathrm{C}_{3} \mathrm{H}_{6} \mathrm{O}\right)$. To demonstrate the robustness of the method, we construct a poor starting determinantal component from a set of unconverged HF orbitals obtained by diagonalizing the Fock matrix built from the Huckel orbitals. A simple electron-electron and electron-nucleus Jastrow factor is employed, and the 473 orbital parameters are optimized by including all 130 virtual orbitals. The starting unconverged eigenvalues are used as estimates for the energy denominators [Eq. (14)]. The SR optimization is performed with the critical value of $\Lambda$ estimated as in Ref. 4 , and converges to the optimal wave function only after 40 steps. The perturbative EFP approach converges in only two steps as the EFP method, with a computational effort as low as the one of the SR method. Interestingly, the perturbative method is successful even when using the eigenvalues of the starting Huckel orbitals to roughly estimate the energies $\Delta E^{i \rightarrow j}$. Finally, if we start from converged HF orbitals, the energy gain from the optimization is of $12 \pm 1$ and $5 \pm 1$ mhartree in VMC and DMC, respectively.

The procedure is not very different if the wave function is constructed from a spin-unrestricted determinant. The variations of an occupied orbital [Eq. (10)] are now with respect to the virtual orbitals of the same spin, and the excitation energies are estimated as $\Delta E^{i, \sigma \rightarrow j, \sigma} \sim \epsilon_{j, \sigma}-\epsilon_{i, \sigma}$ where $\sigma$ is the spin of the varied orbital. The case of a restricted open-shell determinant is instead more complicated since the final orbitals and eigenvalues of a restricted open-shell Hartree-Fock calculation are not uniquely defined. Among the many constructions available in the literature, we find that the one by Guest and Saunders ${ }^{20}$ yields eigenvalues which, on average, well approximate the excitation energies in Eq. (14). Note that closed-to-open-shell excitations must also be included in Eq. (10) to allow full orbital variation.

For a multideterminant wave function from a completeactive-space self-consistent-field (CASSCF) calculation, a set of canonical orbitals is usually defined as diagonalizing the closed, active, and virtual blocks of the so-called generalized Fock matrix. However, to obtain a good estimate of the closed-shell-to-active and active-to-virtual excitation energies, the eigenvalues must be properly adjusted since the energy of an active orbital should be different for excitations into or out of it, i.e., closer to an electron affinity or to an ionization potential, respectively. Therefore, for these excitations, we follow Ref. 21 to define $\Delta E^{i \rightarrow j} \sim E^{i \rightarrow j}-E_{\mathrm{CASSCF}}$ as

$$
\Delta E^{i \rightarrow j} \sim \epsilon_{j}-\epsilon_{i}+\frac{\lambda}{2}\left(\rho_{j j}+2-\rho_{i i}\right)
$$

where $\epsilon$ is a CASSCF eigenvalue, $\rho$ is the single-particle density matrix, and $\lambda$ is an average difference between the electron affinity and the ionization potential of the active orbitals, chosen of the order of $0.3-0.5$ hartree.

Finally, we consider the simultaneous optimization of orbitals and CI coefficients. The simplest approach is to alternate between an orbital optimization step with the perturbative EFP method and a CI calculation in the basis of the CSFs multiplied by the Jastrow factor. ${ }^{8}$ Alternatively, we can use the perturbative EFP method for orbital and CI parameters and sample the quantities in Eq. (4) for both variations. 
TABLE I. VMC and DMC energies in Hartree for the $1{ }^{1} A_{g}$ and the $1^{1} B_{u}$ states of trans-hexatriene. The DMC excitation energies $\Delta E$ are with respect to the ground state obtained with the same wave-function type. We optimize 604 and 493 parameters for the ground and excited states, respectively (Ref. 23). All VMC runs are of $8 \times 10^{5}$ steps. The errors on the last figure are given in parenthesis.

\begin{tabular}{llccc}
\hline \hline State & Wave function & $E_{\mathrm{VMC}}$ & $E_{\mathrm{DMC}}$ & $\begin{array}{c}\Delta E \\
(\mathrm{eV})\end{array}$ \\
\hline${ }^{1}{ }^{1} A_{g}$ & HF & $-38.684(1)$ & $-38.7979(7)$ & \\
& B3LYP & $-38.693(1)$ & $-38.7997(7)$ & \\
& Optimized & $-38.694(1)$ & $-38.7992(7)$ & \\
$1^{1} B_{u}$ & CAS(2,2) & $-38.472(1)$ & $-38.5910(7)$ & $5.63(3)$ \\
& B3LYP & $-38.482(1)$ & $-38.6030(7)$ & $5.35(3)$ \\
& Optimized & $-38.495(1)$ & $-38.6069(8)$ & $5.23(3)$ \\
Expt. ${ }^{\text {a }}$ & & & & 5.22 \\
\hline \hline
\end{tabular}

${ }^{\mathrm{a}}$ Reference 22.

The corrections $V_{k}$ for the CI coefficients are computed in the basis of the CI states obtained in a starting CI calculation, and the energy denominators [Eq. (9)] estimated using the CI energies.

In Table I, we demonstrate the performance of the method on the $1{ }^{1} B_{u}$ state of trans-hexatriene $\left(\mathrm{C}_{6} \mathrm{H}_{8}\right)$ which represents a challenge for all electronic structure approaches: CASPT2 yields a vertical excitation energy of only $5.01 \mathrm{eV}$ (Ref. 24) while the TDDFT energies range between 4.42 and $4.64 \mathrm{eV}$, depending on the functional employed. ${ }^{25}$ For the singledeterminant closed-shell ground state, the VMC energy is improved by about 10 mhartree when otimizing the HF orbitals while, differently from acetone, the DMC energy is not significantly affected. ${ }^{26}$ The excited state is mainly an excitation from the highest occupied (LUMO) to the lowest unoccupied (HOMO) orbital and is well described by a twodeterminant singlet wave function. A CASSCF wave function of two electrons in two orbitals, denoted by CAS(2,2), gives a DMC vertical excitation energy $0.4 \mathrm{eV}$ higher than the experimental value. Employing B3LYP orbitals partially improves the result but only the fully optimized wave function yields an excitation energy in perfect agreement with experiments. Finally, we note that the excited state does not have multiconfigurational character, and using an unoptimized multideterminant wave function yields significantly worse QMC energies: a CASSCF wave function of six electrons in six orbitals, where we keep the leading five CSFs, yields an energy of $-38.438(1)$ and $-38.560(2)$ hartree in VMC and DMC, respectively. Upon optimization of both orbitals and CI coefficients, we recover the best energies of Table I.

We thank C. J. Umrigar for useful discussions. This work is sponsored by the Stichting Nationale Computerfaciliteiten (NCF) for the use of supercomputer facilities.
${ }^{1}$ W. M. C. Foulkes et al., Rev. Mod. Phys. 73, 33 (2001).

${ }^{2}$ C. J. Umrigar, K. G. Wilson, and J. W. Wilkins, Phys. Rev. Lett. 60, 1719 (1988).

${ }^{3}$ With nonlocal pseudopotentials, the full Jastrow-Slater wave function is used to localize the pseudopotential so that the DMC energy will depend on the Jastrow factor.

${ }^{4}$ F. Schautz and C. Filippi, J. Chem. Phys. 120, 10931 (2004).

${ }^{5}$ A. Harju et al., Phys. Rev. Lett. 79, 1173 (1997).

${ }^{6}$ M. Snajdr, J. R. Dwyer, and S. M. Rothstein, J. Chem. Phys. 111, 9971 (1999); 114, 6960(E) (2001).

${ }^{7}$ X. Lin, H. Zhang, and A. M. Rappe, J. Chem. Phys. 112, 2650 (2000); M. W. Lee, M. Mella, and A. M. Rappe, ibid. 122, 244103 (2005).

${ }^{8}$ M. P. Nightingale and V. Melik-Alaverdian, Phys. Rev. Lett. 87, 043401 (2001).

${ }^{9}$ C. J. Umrigar and C. Filippi, Phys. Rev. Lett. 94, 150201 (2005).

${ }^{10}$ S. Sorella, Phys. Rev. B 71, 241103(R) (2005).

${ }^{11}$ S. Sorella, Phys. Rev. B 64, 024512 (2001).

${ }^{12}$ M. Casula and S. Sorella, J. Chem. Phys. 119, 6500 (2003).

${ }^{13} \mathrm{~S}$. Fahy, in Quantum Monte Carlo Methods in Physics and Chemistry, edited by M. P. Nightingale and C. J. Umrigar, NATO Advanced Studies Institute, Series C: Mathematical and Physical Sciences (Plenum, New York, 1999), Vol. 525, p. 101.

${ }^{14}$ C. Filippi and S. Fahy, J. Chem. Phys. 112, 3523 (2000).

${ }^{15}$ F. Schautz and S. Fahy, J. Chem. Phys. 116, 3533 (2002).

${ }^{16}$ D. Prendergast, D. Bevan, and S. Fahy, Phys. Rev. B 66, 155104 (2002).

${ }^{17}$ K. Ruedenberg, L. M. Cheung, and S. T. Elbert, Int. J. Quantum
Chem. 16, 1069 (1997).

${ }^{18}$ In Ref. 4, we modified the ORMAS CI module of the code GAMESS [M. W. Schmidt et al., J. Comput. Chem. 14, 1347 (1993)], where a submatrix of the CI Hamiltonian can be accessed.

${ }^{19}$ Sampling the covariance matrix in Eq. (4) also becomes demanding and a two-step procedure may be adopted: a first run sampling only the derivatives of the energy would be followed by the computation of the covariance matrix for the parameters where the derivative is some standard deviations outside zero.

${ }^{20}$ M. F. Guest and V. R. Saunders, Mol. Phys. 28, 819 (1974).

${ }^{21}$ G. Ghigo, B. O. Roos, and P. A. Malmqvist, Chem. Phys. Lett. 396, 142 (2004).

${ }^{22}$ D. G. Leopold et al., J. Chem. Phys. 81, 4218 (1984). The 0-0 transition to the $1{ }^{1} B_{1 u}$ state is assigned at $4.93 \mathrm{eV}$. Using the excited state vibrational frequencies and displacements, we estimate a Stokes shift of the order of $0.29 \mathrm{eV}$ and a vertical excitation energy of $5.22 \mathrm{eV}$.

${ }^{23}$ To optimize the 17 occupied orbitals in the excited state wave function, we need to include 95 virtual orbitals out of 231 to obtain our best energy.

${ }^{24}$ L. Serrano-Andrés et al., J. Chem. Phys. 98, 3151 (1993).

${ }^{25}$ C.-P. Hsu, S. Hirata, and M. Head-Gordon, J. Phys. Chem. A 105, 451 (2001).

${ }^{26}$ An electron-electron and electron-nucleus Jastrow factor is employed. The Gaussian basis set is $(10 s 10 p 2 d) /[4 s 4 p 2 d]$ for C and $(11 s 2 p) /[4 s 2 p]$ for $\mathrm{H}$. The DMC time step is 0.025 Hartree $^{-1}$. 